\title{
Similarities between Ion Waves in Plasmas and Gravity Waves in Incompressible Fluids
}

\author{
S. H. Lam and Juan R. Sammartin*
}

Gas Dynamics Laboratory, Princeton University, Princeton, New Jersey 08540

(Received 19 June 1970)

\begin{abstract}
Some similarities between ion waves in plasmas and gravity waves in incompressible fluids are investigated. It is shown that for zero ion temperature the ion-wave dispersion relation is similar to that of gravity waves in a stratified liquid between rigid, horizontal walls; for large wavelength the ion waves behave as the surface gravity waves of shallow-water theory. The general character of the pattern of ion waves arising in steady plasma flows is analyzed for arbitrary ion temperature, wavelength, and acoustic Mach number (which is based on the ion-acoustic speed), and is compared to the pattern of surface gravity waves in steady water flows when surface tension is taken into account.
\end{abstract}

\section{INTRODUCTION}

The purpose of this paper is to point out some similarities between gravity waves in incompressible fluids and ion waves in plasmas, and to discuss the general character of the pattern of ion waves in steady plasma flows. ${ }^{1}$ Dissipative effects will be neglected, even though this may be justified only under some restrictive conditions on wavelength, ion temperature and, for weakly ionized plasmas, the ion-neutral collision frequency.

In Secs. II and III, the dispersion relation for ion waves is briefly discussed in the limits of zero ion temperature and large wavelength, respectively. The ion waves are shown to behave as gravity waves in a stratified liquid between rigid, horizontal walls in the first case, and as surface gravity waves in shallow water in the second.

In Sec. IV we consider uniform, steady plasma flows and study in detail the resulting wave pattern for arbitrary wavelength, ion temperature, and acoustic Mach number (the ratio of flow velocity to ion-acoustic speed); the unsteady wave pattern in a plasma at rest may be obtained from the results for large acoustic Mach number. ${ }^{1}$

\section{COLD-ION LIMIT}

To study the electrostatic oscillations of a plasma, one considers Poisson's equation for the electric potential $\phi$,

$$
\hat{\nabla}^{2} \phi=-4 \pi e\left(Z_{i} N_{i}-N_{e}\right)
$$

together with appropriate equations for the ion and electron densities $N_{i}$ and $N_{e}\left(Z_{i}\right.$ is the ion charge number). For the low-frequency (ion) waves discussed in this paper the electrons are in equilibrium with the field so that for small perturbations one may write

$$
N_{e} \approx N_{0}\left(1+e \phi / \kappa T_{e}\right),
$$

where $N_{0}$ and $T_{e}$ are the equilibrium electron density and temperature. For the ions, however, no such simplification is possible. In the zero ion-temperature limit assumed in this section, the ion motion is completely described by continuity and momentum equations,

$$
\begin{aligned}
& \frac{D}{D t} N_{i}+N_{i} \hat{\nabla} \cdot \mathrm{V}_{i}=0, \\
& \frac{D}{D t} \mathrm{~V}_{i}+\frac{Z_{i} e}{m_{i}} \hat{\nabla} \phi=0,
\end{aligned}
$$

where $D / D t \equiv \partial / \partial t+\mathrm{V}_{i} \cdot \hat{\nabla}$, and $m_{i}$ and $\mathrm{V}_{i}$ are the ion mass and mean velocity.

After linearizing Eqs. (3) and (4), the system (1)(4) yields

$$
\left(\lambda_{D}{ }^{2} \frac{\partial^{2}}{\partial t^{2}} \hat{\nabla}^{2}-\frac{\partial^{2}}{\partial t^{2}}+V_{s}^{2} \hat{\nabla}^{2}\right) \phi=0
$$

where $\lambda_{D} \equiv\left(\kappa T_{e} / 4 \pi e^{2} N_{0}\right)^{1 / 2}$ is the electron Debye length and $V_{s} \equiv\left(Z_{i} \kappa T_{e} / m_{i}\right)^{1 / 2}$ is the ion-acoustic velocity (the ion plasma frequency is $\left.\omega_{p i}=V_{s} / \lambda_{D}\right)$. The dispersion relation resulting from (5) is

$$
\omega=V_{s} \hat{k}\left(1+\lambda_{D}{ }^{2} \hat{k}^{2}\right)^{-1 / 2} \text {, }
$$

which represents ion-acoustic waves $\left(\omega \approx V_{s} \hat{k}\right)$ for $\hat{k} \lambda_{D} \ll<1$, and ion plasma waves $\left(\omega \approx \omega_{p i}\right)$ for $\hat{k} \lambda_{D} \gg 1 .^{2}$

Let us now consider an incompressible fluid between two rigid, horizontal walls in a uniform gravity field $\mathrm{g}$. The problem is governed by the incompressibility and continuity equations ${ }^{3}$

$$
\begin{aligned}
& \frac{D}{D t} \rho=0, \\
& \hat{\nabla} \cdot \mathrm{V}=0,
\end{aligned}
$$

together with the momentum equation

$$
\frac{D}{D t} \mathrm{~V}+\rho^{-1} \hat{\nabla} p+g 1_{y}=0 \text {. }
$$

Here, $1_{y}$ is an unit vector along the $y$ axis, which points upward, and $\rho, p$, and $\mathrm{V}$ are the density, pressure, and velócity of the liquid. At rest the liquid is assumed to be stratified according to the law $\rho=\bar{\rho} \equiv \rho_{0} \exp (-\hat{y} / L)$; we then have $\mathrm{V}=0$ and

$$
\frac{\partial p}{\partial \hat{y}}=-\bar{\rho} g \text {, or } p=\bar{p} \equiv g L \bar{\rho} .
$$


For perturbations around this equilibrium we have

$$
\begin{gathered}
\mathrm{V}=\mathrm{v}^{\prime} \equiv \mathrm{v}_{\perp}^{\prime}+w^{\prime} 1_{y}, \\
p=\bar{p}+p^{\prime}, \quad \rho=\bar{\rho}+\rho^{\prime},
\end{gathered}
$$

and linearizing Eqs. (7)-(9) we get

$$
\begin{gathered}
\frac{\partial \rho^{\prime}}{\partial t}+\frac{w w^{\prime} d \bar{\rho}}{d \hat{y}}=0, \\
\hat{\nabla} \perp \cdot \mathbf{v}^{\prime}+\frac{\partial w^{\prime}}{\partial \hat{y}}=0, \\
\frac{\bar{\rho} \partial \mathbf{v}_{\perp}^{\prime}}{\partial t}+\hat{\nabla} \perp p^{\prime}=0, \\
\frac{\bar{\rho} \partial w^{\prime}}{\partial t}+\frac{\partial p^{\prime}}{\partial \hat{y}}-\bar{\rho}-1 \rho^{\prime} \frac{d \bar{p}}{d \hat{y}}=0 .
\end{gathered}
$$

Operating $g \hat{\nabla} \iota^{2}$ on $(10),-(\partial / \partial \hat{y}) \bar{\rho} \partial^{2} / \partial t^{2}$ on (11) $\left(\partial^{2} / \partial t \partial \hat{y}\right) \hat{\nabla}_{\perp}$ on $(12)$, and $-\hat{\nabla}_{\perp}^{2} \partial / \partial t$ on (13), and adding the resulting equations we get

$$
\left(\hat{\nabla}^{2} \frac{\partial^{2}}{\partial t^{2}}+\frac{g}{L} \hat{\nabla}_{\perp}{ }^{2}-L^{-1} \frac{\partial^{2}}{\partial t^{2}} \frac{\partial}{\partial \hat{y}}\right) w^{\prime}=0 .
$$

Assuming that $H \ll L, H$ being the distance between the walls, we may neglect the last term in (14); moreover, $w$ must vanish at $\hat{y}=0$ and $\hat{y}=-H$, so that we have

$$
w^{\prime}=w^{*} \sin \hat{k}_{y} \hat{y}, \quad \hat{k}_{d}=n \pi H^{-1}(n=1,2 \cdots) .
$$

Thus, $w^{*}$ obeys the equation

$$
\left(\frac{H^{2}}{\pi^{2} n^{2}} \frac{\partial^{2}}{\partial t^{2}} \hat{\nabla}_{\perp^{2}}-\frac{\partial^{2}}{\partial t^{2}}+\frac{g H^{2}}{\pi^{2} n^{2} L} \hat{\nabla}_{\perp^{2}}\right) w^{*}=0,
$$

with the dispersion relation

$$
\omega=\left(\frac{g H^{2}}{\pi^{2} n^{2} L}\right)^{1 / 2} \hat{k}\left(1+\frac{H^{2} \hat{k}^{2}}{\pi^{2} n^{2}}\right)^{-1 / 2}
$$

so that the waves from (5) and (15) are entirely similar $\left[H / \pi n\right.$ corresponds to $\lambda_{D}$ and $(g / L)^{1 / 2}$ to $\left.\omega_{p i}\right]$.

\section{LARGE WAVELENGTH LIMIT}

For finite ion temperature Eq. (4) should read

$$
\frac{D \mathrm{~V}_{i}}{D t}+\frac{Z_{i} e}{m_{i}} \hat{\nabla} \phi+\frac{\hat{\nabla} \cdot \mathrm{P}_{i}}{N_{i} m_{i}}=0
$$

where $\mathbf{P}_{i}$ is the pressure tensor. For small perturbations with a wave frequency small compared with the ion-ion collision frequency we may use a scalar pressure that behaves as $N_{i}^{\gamma}$ where $\gamma \equiv(s+2) / s$ is the adiabatic exponent, $s$ being the number of degrees of freedom of the ions; in the opposite limit we may use a scalar pressure that behaves as $N_{i}{ }^{3}$ (corresponding to a onedimensional compression, with $s=1$ in $\gamma$ ), if the phase velocity of the wave is large compared with the ion velocities $^{4}$ (only possible for a small ion-to-electron

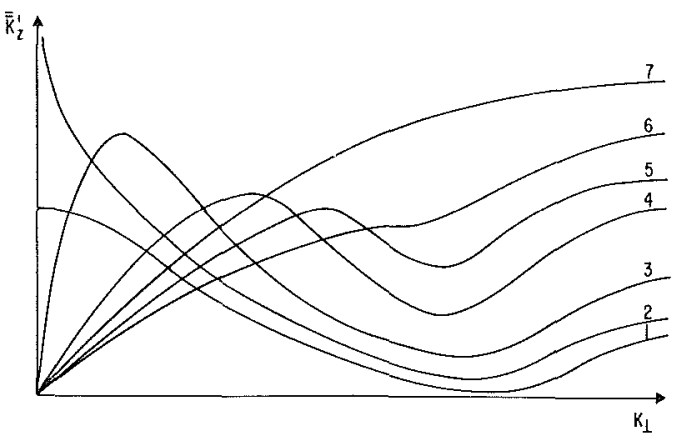

FIG. 1. Schematic representation of $\overline{\bar{k}}_{z}^{\prime}$ vs $k_{\perp}$, for fixed $\epsilon$ and representative values of $M_{s}$.

temperature ratio and wavelengths large compared with the ion Debye length). ${ }^{2}$

After linearization, Eqs. (1) $\sim(3)$ and $\left(4^{\prime}\right)$ yield

$$
\left(-\frac{\gamma \kappa T_{i}}{m_{i}} \lambda_{D}{ }^{2} \hat{\nabla}^{2} \hat{\nabla}^{2}+\lambda_{D}{ }^{2} \frac{\partial^{2}}{\partial t^{2}} \hat{\nabla}^{2}-\frac{\partial^{2}}{\partial t^{2}}+V_{s}{ }^{2} \hat{\nabla}^{2}\right) \phi=0,
$$

where now $V_{s}=\left[\left(Z_{i} T_{e}+\gamma T_{i}\right)_{k} / m_{i}\right]^{1 / 2}$. The dispersion relation is

$$
\omega=V_{8} \hat{k}\left(\frac{1+\epsilon(1+\epsilon)^{-1} \hat{k}^{2} \lambda_{D}^{2}}{1+\lambda_{D}{ }^{2} \hat{k}^{2}}\right)^{1 / 2}, \quad \epsilon=\frac{\gamma T_{i}}{Z_{i} T_{e}}
$$

for $\epsilon \rightarrow 0, V_{s}$ approaches the value given in Sec. II and (6) is recovered from (18). The basic difference between (6) and (18) is that according to (6) the group velocity, $d \omega / d \hat{k}$, decays monotonically to zero, while in (18) $d \omega / d \hat{k}$ passes through a minimum at $\hat{k} \lambda_{D}=$ $\left.\left[1+(4 \epsilon+3)^{1 / 2} \epsilon^{-1 / 2}\right]^{1 / 2}\right\}$ before approaching the limiting value $V_{8} \epsilon^{1 / 2}(1+\epsilon)^{-1 / 2}$; notice that for $\epsilon \ll 1$, the wavelength at the minimum becomes $\lambda_{D}(\epsilon / 3)^{1 / 4} 2 \pi \gg$ $\lambda_{D}(\epsilon / \gamma)^{1 / 2} 2 \pi$ (which is the ion Debye length) so that the aforementioned conditions for the use of the $N_{i}{ }^{3}$ pressure law are satisfied at the minimum.

Let us now consider a nonstratified layer of incompressible fluid in an uniform graviational field $\mathbf{g}$; the fluid has a free-surface and a horizontal bottom at depth $H$. For perturbations with characteristic length, $\lambda$, large compared with $H$, shallow-water theory is applicable and to order $H^{2} \lambda^{-2}$ a standard derivation gives $^{5}$

$$
\left(-\frac{g H^{3}}{6} \hat{\nabla}_{\perp}^{2} \hat{\nabla}_{\perp^{2}}+\frac{H^{2}}{2} \frac{\partial^{2}}{\partial t^{2}} \hat{\nabla}_{\perp^{2}}-\frac{\partial^{2}}{\partial t^{2}}+g H \hat{\nabla}_{\perp^{2}}\right)_{\eta}=0,
$$

where $\eta$ is the deviation of the free surface from its equilibrium and $\hat{\nabla}_{\perp}$ operates in horizontal planes. The dispersion relation is

$$
\omega=(g H)^{1 / 2} \hat{k}\left(\frac{1+H^{2} \hat{k}^{2} / 6}{1+H^{2} \hat{k}^{2} / 2}\right)^{1 / 2}
$$

which is similar to (18) only if $\epsilon=1 / 2$. Remember, however, that (20) is only valid for $H \hat{k} \ll 1$; then, we 


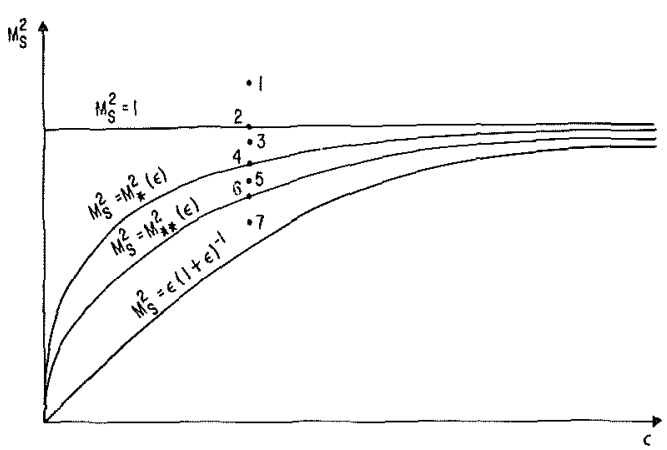

FIg. 2. Relative positions of curves $1-7$ of Fig. 1 in the $M_{s}{ }^{2}-\epsilon$ plane.

have

$$
\omega \approx(g H)^{1 / 2} \hat{k}\left(1-H^{2} \hat{k}^{2} / 3\right)^{1 / 2}
$$

Thus, only the $\lambda_{D} \hat{k} \ll 1$ range in (18) may be compared with (20); for such values Eq. (20) becomes

$$
\omega \approx V_{s} \hat{k}\left[1-(1+\epsilon)^{-1} \lambda_{D} \hat{k}^{2}\right]^{1 / 2}
$$

which is similar to $\left(20^{\prime}\right)$ for any $\epsilon\left[(g H)^{1 / 2}\right.$ corresponds to $V_{s}$ and $3^{-1 / 2} H$ to $\left.\lambda_{D}(1+\epsilon)^{-1 / 2}\right]$. Equation $\left(20^{\prime}\right)$ could be directly obtained from the dispersion relation for surface gravity waves in water of arbitrary depth, ${ }^{6}$

$$
\omega=(g \hat{k})^{1 / 2}(\tanh \hat{k} H)^{1 / 2},
$$

by expanding (21) for small $\hat{k} H$.

\section{STEADY WAVE PATTERNS IN UNIFORM FLOWS}

In this section we study the wave patterns arising from (18) for arbitrary $\epsilon$ and $\hat{k} \lambda_{D}$, and compare it with the results of water-wave theory (when both gravity and surface tension effects are considered). Although there is no complete similarity such as in the limiting cases $\epsilon \rightarrow 0$ or $\hat{k}_{D} \lambda \ll 1$, most essential features in both problems appear to be the same.

We consider steady-state waves in uniform flows and introduce the acoustic Mach number, $M_{s} \equiv U / V_{s}$ (where $U$ is the flow velocity), referring to superacoustic and subacoustic flows for $M_{s}>1$ and $M_{s}<1$, respectively; the unsteady wave pattern in a plasma at rest may be obtained from the limit $M_{s} \rightarrow \infty$ (hyperacoustic equivalence principle ${ }^{1}$ ). The analysis of Ref. 1 was restricted to rarefied plasmas, for which the large phase-velocity condition of Sec. III must be satisfied; this condition requires $\epsilon \ll<1$ and $M_{s}^{2} \gg \epsilon$. In Ref. 1, however, it was assumed that $M_{s}{ }^{2}>4^{4 / 3} 3^{-1} \epsilon^{1 / 3}$, and this restriction is now removed. The present analysis will cover the entire range of $\epsilon, M_{s}^{2}$, and $\hat{k} \lambda_{D}$.

Equation (17) only holds at large distances from the source of perturbation; moreover, only at such distances does a behavior develop which is essentially independent of the source. Thus, our analysis will only deal with the asymptotic character of the wave pattern.

A steady-state equation may be obtained from (17) by using the transformation $\partial / \partial t \rightarrow U \partial / \partial \hat{z}$, where $\hat{z}$ is along the direction of the flow. We have

$\left(-\frac{\epsilon}{1+\epsilon} \nabla^{2} \nabla^{2}+M_{s}^{2} \frac{\partial^{2}}{\partial z^{2}} \nabla^{2}-M_{s}{ }^{2} \frac{\partial^{2}}{\partial z^{2}}+\nabla^{2}\right) \phi=0$,

where $\nabla=\lambda_{D} \hat{\nabla}$ and $\nabla^{2} \equiv \partial^{2} / \partial z^{2}+\nabla_{\perp}^{2} ;(22)$ gives

$$
k_{z} M_{s}=k\left[1+\epsilon(1+\epsilon)^{-1} k^{2}\right]^{1 / 2}\left(1+k^{2}\right)^{-1 / 2},
$$

where $k \equiv\left(k_{z}{ }^{2}+k_{\perp}{ }^{2}\right)^{1 / 2}=\hat{k} \lambda_{D}$. Equation (23) has four roots

$$
k_{z}= \pm i \vec{k}_{z}, \quad k_{z}= \pm \bar{k}_{z}
$$

$\bar{k}_{z}$ is always real and yields elliptic behavior for $\phi$; this first couple of roots is due to the fact that in (22) z acts both as a timelike variable and as a space coordinate. For $M_{s}^{2}<\epsilon(1+\epsilon)^{-1}, \overline{\bar{k}}_{z}$ is imaginary and thus the entire behavior of $\phi$ is elliptic; no waves are excited. On the other hand, for $M_{s}^{2}>\epsilon(1+\epsilon)^{-1}, \bar{k}_{z}$ is real and therefore the second couple of roots in (24) yield hyperbolic behavior for $\phi$. The resulting waves are, of course, ion waves.

It may seem surprising that the critical value of $M_{\mathrm{s}}{ }^{2}$ is $\epsilon(1+\epsilon)^{-1}$ and not unity. This may be understood by noticing that as $\hat{k}$ increases, the phase velocity, $\omega / \hat{k}$, decays monotonically from $V_{s}$ to $V_{s} \epsilon^{1 / 2}(1+\epsilon)^{-1 / 2}$. For $U>V_{s}\left(M_{s}>1\right)$ the whole $\hat{k}$ spectrum in (18) is excited since the flow is "supersonic" for all $\hat{k}$; for $V_{s}>U>V_{s} \epsilon^{1 / 2}(1+\epsilon)^{-1 / 2}$, only part of the spectrum may be observed [the range $\hat{k} \geq K, K$ being given by $\omega(K) / K=U]$. For $U<V_{s} \epsilon^{1 / 2}(1+\epsilon)^{-1 / 2}\left[M_{s}^{2}<\right.$ $\left.\epsilon(1+\epsilon)^{-1}\right]$, no waves can be observed because the flow is "subsonic" for all $\hat{k}$.

At large distances, the contribution of the $\overline{\bar{k}}_{z}$ roots becomes dominant; $\overline{\bar{k}}_{z}\left(k_{\perp}, \epsilon, M_{s}\right)$ is given below as a function of $k_{\perp}, \epsilon$ and $\tau \equiv\left[(1+\epsilon) \epsilon^{-1} M_{s}^{2}-1\right]^{-1}$ :

$$
\begin{aligned}
\overline{\bar{k}}_{z}=2^{-1 / 2}\left\{\left[\left(k_{\perp}{ }^{2}(1+\tau)+\frac{\epsilon+\tau}{\epsilon}\right)^{2}-\right.\right. & \left.\frac{4 \tau}{\epsilon}\right]^{1 / 2} \\
& \left.+k_{\perp}^{2}(\tau-1)+\frac{\tau-\epsilon}{\epsilon}\right\}^{1 / 2} .
\end{aligned}
$$

Of particular importance for the asymptotic wave pattern is the behavior of $\overline{\bar{k}}_{z}{ }^{\prime} \equiv \partial \overline{\bar{k}}_{z} / \partial k_{\perp}$; this is schematically represented in Fig. 1 for a given $\epsilon$ and representative values of $M_{s}^{2}$. (The relative positions of curves $1-7$ in the parametrical $M_{s}^{2}-\epsilon$ plane are indicated in Fig. 2). The appearance of maxima and minima in Fig. 1 is of special interest because the usual asymptotic analysis based on the group-velocity concept (stationary-phase method) shows that steep wave fronts develop at values of $\sigma(\sigma \equiv x / z, x$ being the transverse space coordinate) coinciding with any of those extremal values of $\bar{k}_{z}{ }^{\prime},{ }^{7}$ For reference we give 
TABLE I. Values of $\sigma_{3}\left[\epsilon, M_{* *}{ }^{2}(\epsilon) \leq M_{8}^{2}<\infty\right]$.

\begin{tabular}{|c|c|c|c|c|c|c|c|}
\hline $\boldsymbol{\epsilon} \ll 1$ & $\begin{array}{c}M_{8}^{2} \\
\sigma_{3}\end{array}$ & $\begin{array}{c}M_{* *^{2}}(\epsilon) \sim 2 \epsilon^{1 / 2} \\
\epsilon^{1 / 2}\end{array}$ & \multicolumn{4}{|c|}{$\begin{array}{c}M_{s}{ }^{2}=O\left(\epsilon^{1 / 2}\right) \\
\epsilon^{1 / 2}(4 x)^{-1}\left(1+x^{2}\right)^{3 / 2}\left(3-x^{2}\right)^{1 / 2} a\end{array}$} & $\begin{array}{c}M_{s}^{2} \gg \epsilon^{1 / 2} \\
1.75 \epsilon^{3 / 4} M_{s}^{-1}\end{array}$ \\
\hline$\epsilon=0.104$ & $\begin{array}{l}M_{8}^{2} \\
\sigma_{3}\end{array}$ & $\begin{array}{l}0.470 \\
0.322\end{array}$ & $\begin{array}{l}0.586 \\
0.300\end{array}$ & $\begin{array}{l}1.05 \\
0.229\end{array}$ & $\begin{array}{l}1.36 \\
0.203\end{array}$ & $\begin{array}{l}2.09 \\
0.163\end{array}$ & \multirow{4}{*}{$\begin{array}{c}M_{s}^{2} \gg 1 \\
\sigma_{3}=1.15 \epsilon^{1 / 2}(1+\epsilon)^{-1 / 2} M_{s}^{-1} \\
\times \frac{\left[1+(4+3 / \epsilon)^{1 / 2}\right]^{1 / 2}}{1+(4+3 / \epsilon)^{1 / 2} 2^{-1}}+O\left(M_{s}^{-3}\right)\end{array}$} \\
\hline$\epsilon=0.770$ & $\begin{array}{l}M_{8}^{2} \\
\sigma_{3}\end{array}$ & $\begin{array}{l}0.795 \\
0.877\end{array}$ & $\begin{array}{l}0.811 \\
0.870\end{array}$ & $\begin{array}{l}1.04 \\
0.728\end{array}$ & $\begin{array}{l}1.84 \\
0.505\end{array}$ & & \\
\hline$\epsilon=3.31$ & $\begin{array}{l}M_{8}^{2} \\
\sigma_{3}\end{array}$ & $\begin{array}{l}0.934 \\
1.82\end{array}$ & $\begin{array}{l}0.967 \\
1.73\end{array}$ & $\begin{array}{l}1.60 \\
0.925\end{array}$ & $\begin{array}{l}2.29 \\
0.691\end{array}$ & & \\
\hline$\epsilon=10.0$ & $\begin{array}{l}M_{3}^{2} \\
\sigma_{3}\end{array}$ & $\begin{array}{l}0.975 \\
3.16\end{array}$ & $\begin{array}{l}0.995 \\
2.95\end{array}$ & $\begin{array}{l}1.84 \\
0.970\end{array}$ & & & \\
\hline$\Leftrightarrow \gg 1$ & $\sigma_{3}=$ & $\frac{0.433}{\left.1+\epsilon) \epsilon^{-1}-1\right]^{1 / 2}}$ & $\frac{2+\{4-}{1+\{4-3}$ & $\begin{array}{l}\frac{c}{6}\left(M_{8}^{2}-\right. \\
\left(M_{s}^{2}-1\right.\end{array}$ & $\frac{\left.+1]^{-1}\right\}}{\left.+1]^{-1}\right\}^{1}}$ & & \\
\hline
\end{tabular}

$x=\cos (\theta / 3)+3^{1 / 2} \sin (\theta / 3), \theta=\cos ^{-1}\left(2 \epsilon^{1 / 2} M_{s}^{-2}\right)$.

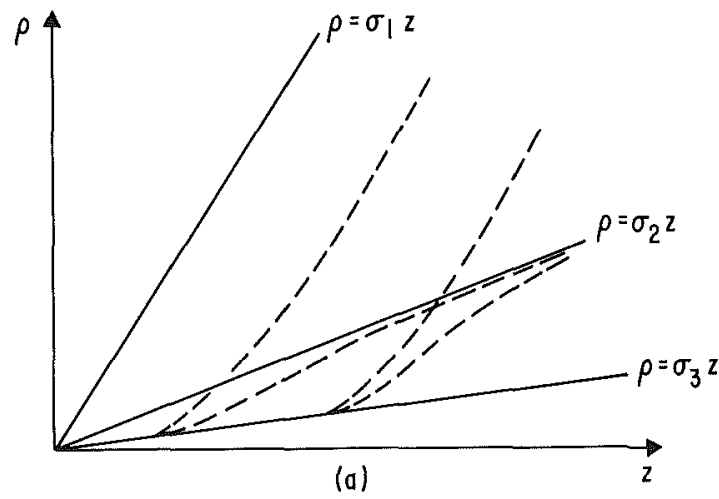

(a)

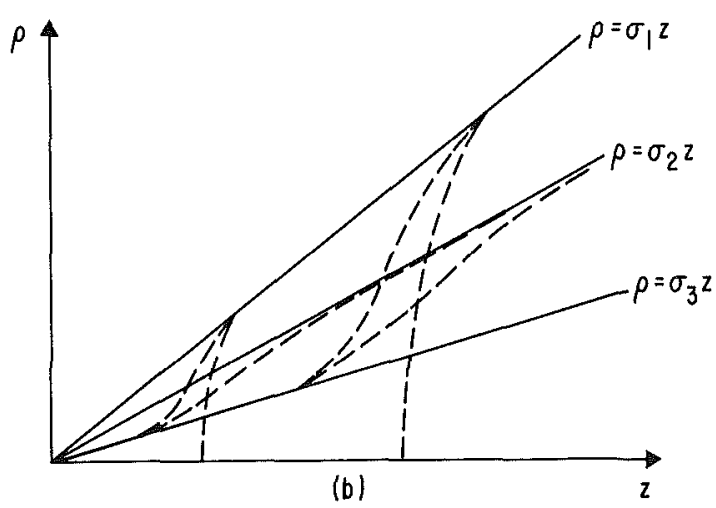

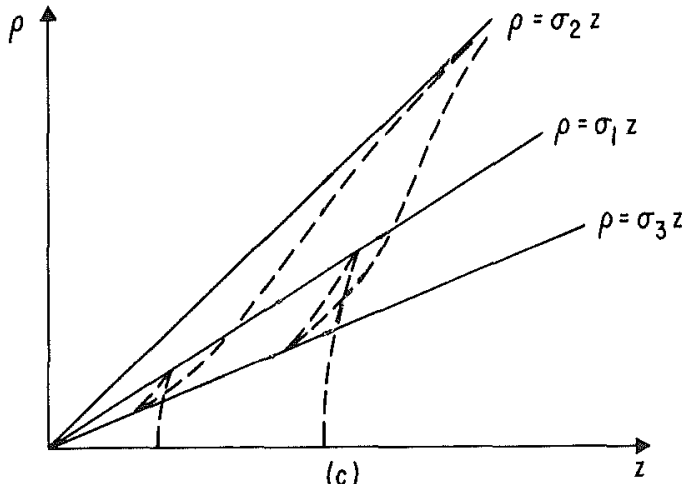

(c)

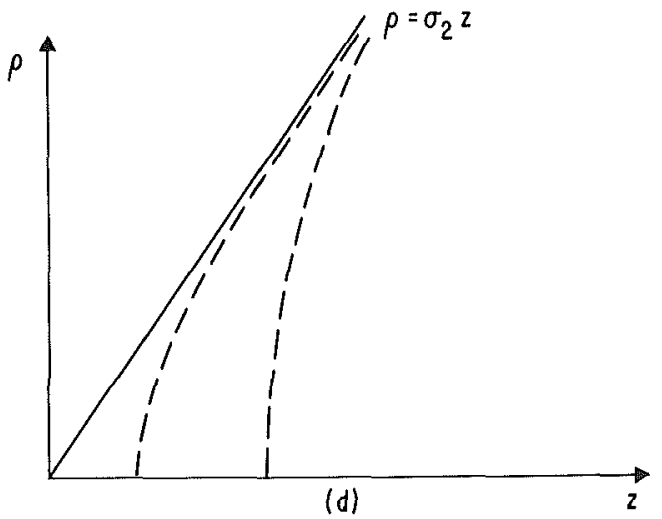

FIG. 3. Schematic representation of ion-wave patterns (- wave fronts, - - curves of constant phase); perturbation localized at the origin. (a) $1<M_{s}^{2}<\infty$; (b) $M *^{2}<M_{s}{ }^{2}<1$; (c) $M * *^{2}<M_{s}{ }^{2}<M *^{2}$; (d) $\epsilon(1+\epsilon)^{-1}<M_{s}^{2}<M * *^{2}$. 
TABLE II. Values of $\sigma_{1}\left[\epsilon, M_{* * *}{ }^{2}(\epsilon) \leq M_{s}{ }^{2} \leq 1\right]$

\begin{tabular}{|c|c|c|c|c|c|c|c|c|}
\hline \multirow{2}{*}{$\begin{array}{l}\epsilon \ll 1 \\
\epsilon=0.104\end{array}$} & \multirow{2}{*}{$\begin{array}{l}M_{8}{ }^{2} \\
\boldsymbol{g}_{1} \\
M_{r_{i}}{ }^{2} \\
\sigma_{1}\end{array}$} & \multirow{2}{*}{ 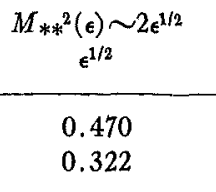 } & \multicolumn{3}{|c|}{$\begin{array}{c}M_{e}^{2}=O\left(\epsilon^{1 / 2}\right) \\
\epsilon^{1 / 2}(4 x)^{-1}\left(1+x^{2}\right)^{3 / 2}\left(3-x^{2}\right)^{1 / 2}\end{array}$} & \multicolumn{3}{|c|}{$\begin{array}{c}M_{s}{ }^{2} \gg s^{1 / 2} \\
2^{-1 / 2}\left(1-M_{s}^{2}\right)^{-1 / 2}\left[\left(1-\frac{3}{4} M_{s}^{2}\right)^{3 / 2}-1+\frac{9}{8} M_{s}{ }^{2}\right]^{1 / 2}\end{array}$} \\
\hline & & & $\begin{array}{l}0.514 \\
0.331\end{array}$ & $\begin{array}{l}0.641 \\
0.414\end{array}$ & $\begin{array}{l}0.808 \\
0.652\end{array}$ & $\begin{array}{l}0.895 \\
0.958\end{array}$ & $\begin{array}{l}0.993 \\
4.53\end{array}$ & \multirow{4}{*}{$\begin{array}{l}\sigma_{1}=8^{-1 / 2}\left(1-M_{s}^{2}\right)^{-1 / 2} \\
\quad \times\left[1-\left(1-M_{8}^{2}\right)(1-\epsilon) 9 / 8\right] \\
+O\left(\left[1-M_{s}^{2}\right]^{3 / 2}\right)\end{array}$} \\
\hline$\epsilon=0.770$ & $\begin{array}{l}M_{s}^{2} \\
\sigma_{1}\end{array}$ & $\begin{array}{l}0.795 \\
0.877\end{array}$ & $\begin{array}{l}0.870 \\
1.00\end{array}$ & $\begin{array}{l}0.910 \\
1.18\end{array}$ & $\begin{array}{l}0.992 \\
4.08\end{array}$ & & & \\
\hline$\epsilon=3,31$ & $\begin{array}{l}M_{\theta}^{2} \\
\sigma_{1}\end{array}$ & $\begin{array}{l}0.934 \\
1.82\end{array}$ & $\begin{array}{l}0.961 \\
2.01\end{array}$ & & & & & \\
\hline$\epsilon=10.0$ & $\begin{array}{l}M_{8}^{2} \\
\sigma_{1}\end{array}$ & $\begin{array}{l}0.975 \\
3.16\end{array}$ & $\begin{array}{l}0.985 \\
3.45\end{array}$ & & & & & \\
\hline$\Leftrightarrow \gg 1$ & $\sigma_{1}$ & $\frac{0.433}{\left[M_{s}^{2}(1+\epsilon) \epsilon\right.}$ & $\frac{1-1]^{1 / 2}}{1}$ & $\frac{2-\{4-3}{1-|4-3|}$ & $\frac{\left(M_{s}^{2}-1\right)}{\left(M_{s}^{2}-1\right)}$ & $\frac{\left.+1]^{-1}\right\}^{1 / 2}}{\left.\left.-1]^{-1}\right\}^{1 / 2}\right)}$ & & \\
\hline
\end{tabular}

${ }^{a} x=\cos (\theta / 3)-3^{1 / 2} \sin (\theta / 3), \theta=\cos ^{-1}\left(2 \epsilon^{1 / 2} M_{s}{ }^{-2}\right)$.

simple expressions for both $\overline{\bar{k}}_{z}{ }^{\prime}$ and ${\overline{k_{z}}}^{\prime \prime} \equiv \partial^{2}{\overline{k_{z}}}_{z} / \partial k_{\Lambda^{2}}$ :

$$
\begin{gathered}
\overline{\bar{k}}_{z}{ }^{\prime}=\frac{\epsilon s^{2}+1}{\epsilon s^{2}-\tau}\left(\frac{\epsilon s-\tau}{\epsilon s+1}\right)^{1 / 2} \tau^{1 / 2}, \\
\overline{\bar{k}}_{z}^{\prime \prime}=\frac{\epsilon^{7 / 2} s^{3 / 2}(s-1)^{1 / 2} \tau^{1 / 2}(\tau+1)^{3 / 2}}{(\epsilon s+1)^{3 / 2}\left(\epsilon s^{2}-\tau\right)^{3}} \\
\times\left(s^{3}(s-4)-\frac{3 s^{2}}{\epsilon}(1-\tau)+\tau \frac{4 s-1}{\epsilon^{2}}\right),
\end{gathered}
$$

where

$$
s \equiv k_{\perp}^{2}+1+\overline{\bar{k}}_{z}^{2}
$$

$$
\left[k_{\perp}=(s-1)^{1 / 2}(\epsilon s-\tau)^{1 / 2}(\epsilon s)^{-1 / 2}(\tau+1)^{-1 / 2}\right] .
$$

For $1<M_{s}{ }^{2}<\infty$ (curve 1 in Fig. 1) there are maxima at $k_{\perp}=0$ and at $k_{\perp}=\infty$; the first maximum is

$$
\overline{\bar{k}}_{z}^{\prime}=\sigma_{1} \equiv\left(M_{s}{ }^{2}-1\right)^{-1 / 2} \text {, }
$$

and the second one

$$
\bar{k}_{z}{ }^{\prime}=\sigma_{2} \equiv\left[(1+\epsilon) \epsilon^{-1} M_{s}{ }^{2}-1\right]^{-1 / 2} .
$$

There is also a minimum, $\overline{\bar{k}}_{z}{ }^{\prime}=\sigma_{3}$, at a finite $k_{\perp} ; \sigma_{3}$ is given in Table I as a function of $\epsilon$ and $M_{\mathrm{s}}{ }^{2}$. As $M_{\mathrm{s}}{ }^{2} \rightarrow \infty$, all three $\sigma_{1}, \sigma_{2}$, and $\sigma_{3}$ go to zero as $M_{s}^{-1}$; the behavior of $\sigma_{3}$ is

$$
\sigma_{3} \approx \frac{1.15 \epsilon^{1 / 2}\left[1+(4+3 / \epsilon)^{1 / 2}\right]^{1 / 2}}{(1+\epsilon)^{1 / 2}\left[1+(1+3 / 4 \epsilon)^{1 / 2}\right] M_{8}} .
$$

As $M_{s}{ }^{2} \rightarrow 1$ (curve 2), $\sigma_{1} \rightarrow \infty, \sigma_{2} \rightarrow \epsilon^{1 / 2}$, and

$$
\sigma_{3} \approx \epsilon^{-1 / 2} \frac{(8 / 3)^{1 / 2}\left[1+(1+4 / 3 \epsilon)^{1 / 2}\right]^{-1}+(27 / 8)^{1 / 2} \epsilon}{\left[5+3(1+4 / 3 \epsilon)^{1 / 2}\right]\left[1+3(1+4 / 3 \epsilon)^{1 / 2}\right]^{1 / 2}} .
$$

As $M_{s}{ }^{2}$ decreases below 1 (curve 3), $\sigma_{1}$ falls down and moves away from $k_{\perp}=0 ; \sigma_{1}$ is now a complicated function of $M_{s}{ }^{2}$ and $\epsilon$, given in Table II. $\sigma_{2}$ is still given by (25) and $\sigma_{3}$ by Table I.
As $M_{s}{ }^{2}$ decreases, we reach a value, $M_{\mathrm{s}}{ }^{2}=M_{*}{ }^{2}(\epsilon)$ (curve 4), for which $\sigma_{1}=\sigma_{2}$. Both $M *^{2}$ and the common value of $\sigma_{1}$ and $\sigma_{2}$ at this value of $M_{s}^{2}, \sigma_{*}$, are given in Table III, as functions of $\epsilon$.

For smaller $M_{s}{ }^{2}$ (curve 5) $\sigma_{2}$ is the largest maximum.

If we decrease $M_{s}{ }^{2}$ still further we reach a value, $M_{8}{ }^{2}=M_{* *}{ }^{2}(\epsilon)$ (curve 6) for which $\sigma_{1}=\sigma_{3} ;$ both extremes coalesce giving $\bar{k}_{z}^{\prime \prime \prime}=0$. We have

$$
M_{* *}(\epsilon) \equiv 2\left[\left(\epsilon+\epsilon^{2}\right)^{1 / 2}-\epsilon\right],
$$

$$
\sigma * *(\epsilon)=\epsilon^{1 / 2},
$$

$\sigma_{* *}$ being the common value of $\sigma_{1}$ and $\sigma_{3}$.

Finally, for $M_{8}^{2}<M_{* *}{ }^{2}(\epsilon)$, there is only one maximum, $\sigma_{2}$ (curve 7). As $M_{\varepsilon}{ }^{2} \rightarrow \epsilon(1+\epsilon)^{-1}, \sigma_{2} \rightarrow \infty$ and for smaller $M_{s}^{2}, \bar{k}_{s}$ is purely imaginary.

From these results some general features of the wave

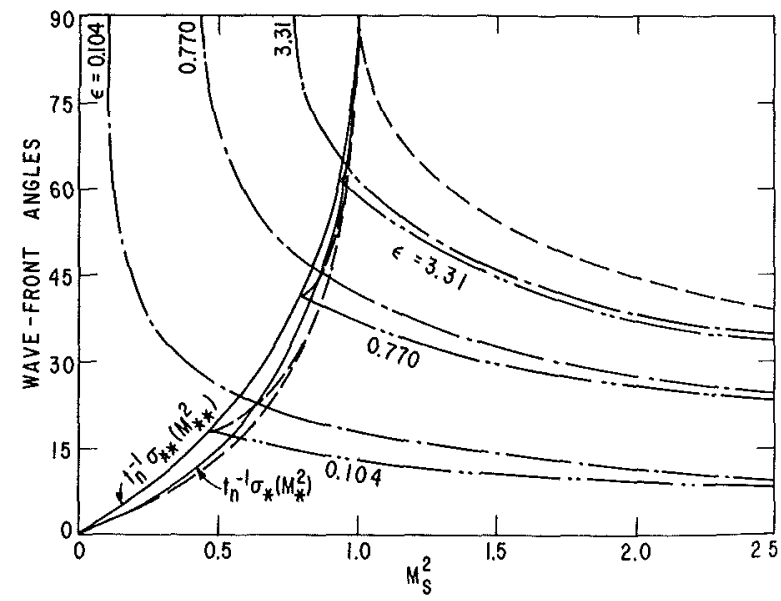

FIG. 4. Wave-front angles vs $M_{s}^{2}$ and $\epsilon\left(--\tan ^{-1} \sigma_{1},-\cdot-\tan ^{-1} \sigma_{2}\right.$ $\left.-\cdots-\tan ^{-1} \sigma_{3}\right)$. 
TABLE III. Values of $M_{*}{ }^{2}(\epsilon)$ and $\sigma_{*}(\epsilon)$.

\begin{tabular}{ccccccccc}
\hline$\epsilon$ & $\epsilon \ll 1$ & 0.0144 & 0.104 & 0.770 & 3.31 & 10.0 & $\epsilon \gg 1$ \\
\hline$M_{*}^{2}$ & $2.11 \epsilon^{1 / 3}+O\left(\epsilon^{2 / 3}\right)$ & 0.406 & 0.641 & 0.870 & 0.961 & 0.985 & $1-0.156 \epsilon^{-1}+O\left(\epsilon^{-2}\right)$ \\
$\sigma *$ & $0.687 \epsilon^{1 / 3}+O\left(\epsilon^{2 / 3}\right)$ & 0.191 & 0.414 & 1.00 & 2.01 & 3.45 & $1.08 \epsilon^{1 / 2}+O\left(\epsilon^{-1 / 2}\right)$ \\
\hline
\end{tabular}

pattern may easily be derived through the use of the group-velocity concept ${ }^{7}$ (for details, see Ref. 1). For $M_{\varepsilon}>M_{*}(\epsilon)$ the maximum "group velocity" is $\sigma_{1}$; for $\sigma>\sigma_{1}$ the plasma is, therefore, quasineutral (no waves). For $M_{s}<M *(\epsilon)$, the plasma is quasineutral for $\sigma>\sigma_{2}$. The wave pattern is schematically represented in Fig. $3(\mathrm{a}) \rightarrow(\mathrm{d})$ where we notice that several sets of waves appear. The curves of constant phase are given for each set. For $1<M_{B}<\infty$ there are three wave fronts at $\sigma=\sigma_{1}, \sigma_{2}$, and $\sigma_{3}$; the plasma is quasineutral behind the $\sigma_{3}$ front, sustains two sets of waves between $\sigma_{2}$ and $\sigma_{2}$, and one set between $\sigma_{1}$ and $\sigma_{2}$.

As $M_{3} \rightarrow \infty$ all three fronts collapse into the $z$ axis, retaining the topological order of Fig. 3(a). As $M_{8} \rightarrow 1$, the leading front approaches the $x$ axis.

When $M_{s}$ falls below 1 , this front bounces back approaching the $\sigma_{2}$ front; a new type of wave (transverse) appears on all three regions behind $\sigma=\sigma_{1}$ (the region near the axis is no longer quasineutral).

For $M_{s}<M_{*}(\boldsymbol{\epsilon}), \sigma_{2}$ becomes the leading front and there is only one set of waves between $\sigma_{2}$ and $\sigma_{3}$. Notice that $\sigma *$ is the minimum value of $\max \left(\sigma_{1}, \sigma_{2}\right)$ over the range $M_{s}<1$; thus in subacoustic flow there is a minimum value of the angle of the leading front. This minimum depends on $\epsilon$ and goes to zero as $\epsilon \rightarrow 0$. $\operatorname{Max}\left(\sigma_{1}, \sigma_{2}\right)$ is given in Fig. 4 as a function of $\epsilon$ and $M_{\mathrm{s}}{ }^{2}$.

At $M_{s}=M_{* *}$, the wave front at $\sigma=\sigma * *$ is particularly strong. When $M_{s}<M_{* *}(\epsilon)$, there is only one wavefront and one set of waves. As $M_{s} \rightarrow \epsilon(1+\epsilon)^{-1}$ this front approaches the $x$ axis and all waves disappear for smaller $M_{s}$.

Now consider the dispersion relation for water waves when both gravity and surface tension effects are taken into account, ${ }^{6}$

$$
\omega=(g \hat{k} \tanh \hat{k} H)^{1 / 2}\left[1+(T / \rho g) \hat{k}^{2}\right]^{1 / 2} .
$$

For steady waves in an uniform flow at speed $U$, we define $k=\hat{k} H, T\left(\rho g H^{2}\right)^{-1}=\epsilon, M_{s}=U(g H)^{-1 / 2}$ and obtain

$$
M_{s} k_{z}=(k \tanh k)^{1 / 2}\left(1+\epsilon k^{2}\right)^{1 / 2},
$$

which may be compared with (23).
We notice the following common features of Eqs. (18) and (26): (1) As $\hat{k} \rightarrow 0, \omega \sim \hat{k}$. (2) $d \omega / d \hat{k}$ has a relative (and absolute) minimum [in Eq. (26), only if $\epsilon<3^{-1}$; this will be assumed here]. (3) $\omega / \hat{k}$ has a minimum [at finite $\hat{k}$ in (26), at $\hat{k}=\infty$ in (18)]. (4) For $\epsilon=0, d \omega / d \hat{k}$ decays monotomically to zero as $\hat{k}$ increases.

The only basic difference between (18) and (26) is that as $\hat{k} \rightarrow \infty, \omega \sim \hat{k}$ in the first equation while $\omega \sim \hat{k}^{3 / 2}$ in the second; thus, there is no absolute maximum of the group-velocity in (26). For steady flows, however, only those waves that satisfy $\omega / \hat{k}<U$ are observed so that an effective maximum of $d \omega / d \hat{k}$ exists in (26) too.

From the preceding discussion one may easily conclude that the general features derived from (23) for steady ion waves may be derived from (27) for water waves. In particular, curves in the parametrical $M_{\mathrm{s}}{ }^{2}-\epsilon$ plane of (27) exist (equivalent to those of Fig. 2) such that, if crossed, essential modifications of the wave pattern result; the different wave patterns are similar to those of Fig. 3. (See end of Art. 272 and the figure in page 470 of Ref. 8.)

\section{ACKNOWLEDGMENT}

The authors acknowledge support from the National Aeronautics and Space Administration, Grant NGR 31-001-103.

* Present address: Mechanical Engineering Department, Massachusetts Institute of Technology, Cambridge, Massachusetts 02139 .

1J. R. Sanmartin and S. H. Lam, Phys. Fluids 14, 62 (1971).

2 A. I. Akhiezer, I. A. Akhiezer, R. V. Polovin, A. G. Sitenko, and K. N. Stepanov, Collective Oscillations in a Plasma, translated by H. S. H. Massey (MIT Press, Cambridge, Mass., 1967), p. 22. ${ }^{3}$ C. S. Yih, Dynamics of Nonhomogeneous Fluids (MacMillan, New York, 1965), Chap. 1.

${ }^{4}$ I. B. Bernstein and S. K. Trehan, Nucl. Fusion 1, 3 (1960). ${ }^{5}$ L. J. F. Broer, Appl. Sci. Res. B11, 273 (1963).

$6 \mathrm{~J}$. V. Wehausen and E. V. Laitone, in Handbuch der Physik, edited by S. Flügge (Springer-Verlag, Berlin, 1960), Vol. IX, p. 514.

${ }_{7}^{7}$ H. Jeffreys and B. S. Jeffreys, Methods of Mathematical Physics (Cambridge University Press, London, 1950), Chap. 17.

${ }^{8} \mathrm{H}$. Lamb, Hydrodynamics (Dover, New York, 1945), Chap. IX 